\title{
DINÁMICA DE LAS VOCALES DEL ESPAÑOL EN CONTACTO CON EL CATALÁN
}

\author{
MARÍA J. MACHUCA AYUSO \\ DOLORS POCH OLIVÉ \\ Universidad Autónoma de Barcelona
}

\section{RESUMEN}

Este trabajo estudia las características acústicas de las vocales del español realizadas por hablantes bilingües de catalán y español, cuyo uso de ambas lenguas es distinto, con el objetivo de verificar el peso de la lengua más utilizada en las características de las vocales pronunciadas. Los datos proceden de dos hablantes cuyo porcentaje de utilización del español es mucho mayor que el del catalán, dos hablantes que presentan la situación opuesta y dos hablantes cuyo empleo de ambas lenguas es similar. En todos los casos se ha analizado un corpus de lista de palabras y un corpus de habla espontánea con el fin de estudiar la dinámica global a la que está sometido el sistema vocálico en función del estilo de habla en el que se expresan los informantes.

PAlabRas ClaVE: Fonética acústica, bilingüismo, español, catalán.

\section{ABSTRACT}

The aim of this work is to determine the relationship between the acoustic characteristics of the Spanish vowels pronounced by Catalan and Spanish bilingual speakers and the fact that the speakers use both languages with a different percentage. Six speakers have been recorded: two of them who speak more Spanish than Catalan in their daily life, another pair of speakers who speak more Catalan than Spanish and, finally, another pair of speakers who use both languages, Catalan and Spanish, in a similar way. The first two formants of the Spanish vowels have been analysed in a corpus of spontaneous speech and in a list of words in order to study if the acoustic characteristics of the vowels depend on the style of speech.

Keywords: Acoustic Phonetics, bilingualism, Spanish, Catalan.

\section{INTRODUCCIÓN}

Durante la segunda mitad del siglo xx son muy numerosos los estudios sobre la interferencia entre español y catalán, y la mayoría de ellos com- 
parte la característica común de abordar la cuestión del contacto de estas lenguas desde una perspectiva sociolingüística. Así lo demuestran Sinner y Wesch (2008), cuyo objetivo es presentar un estado de la cuestión de las investigaciones más importantes realizadas sobre el castellano de Cataluña hasta la fecha de publicación de la obra.

En su trabajo, estos autores destacan (págs. 15-17), en primer lugar, los estudios pioneros en este campo: Moll (1961), Badia Margarit (1955, 1965 y 1979) y Colón (1967). El trabajo de Moll (1961) es el primero que hace hincapié en que los catalanismos en español no constituyen un dominio de las clases incultas y en que las particularidades del castellano catalanizado ya se adquieren durante el proceso de aprendizaje de la lengua materna. Los estudios de Badia Margarit (1955, 1965 y 1979) establecen las diferentes etapas del contacto entre las dos lenguas desde el inicio de las grandes oleadas de inmigrantes procedentes del sur de España en la década del cincuenta hasta la época de la normalización del catalán. Finalmente, el artículo de Germán Colón (1967) distingue entre el español hablado por los nativos catalanes, como lengua habitual o preferida por algunos o como lengua ocasional o impuesta por los más, y el español hablado por los inmigrantes y por sus descendientes.

Señalan a continuación Sinner y Wesch (2008: 19) que en la década de 1980 ven la luz los trabajos de Cerdà (1984), Payrató (1985) y Marsá (1986), que inician el análisis de los fenómenos de interferencia. Este tipo de trabajos conocerá un gran auge a partir de los noventa, época en la que se abre un gran debate sobre el concepto mismo de interferencia y comienza a forjarse la idea de que existe un modelo de castellano hablado por catalanohablantes que constituye una variedad específica de dicha lengua (Sinner y Wesch, 2008: 19). En este marco se inserta el volumen de Sinner (2004) que proporciona una descripción de las características léxicas y morfosintácticas del español hablado en Cataluña y, sobre todo, un modelo del funcionamiento de dicha variedad que contempla el tipo de relaciones que mantienen las dos lenguas utilizadas en este territorio.

Como se ha indicado, la mayoría de estudios son de carácter sociolingüístico y los que analizan los fenómenos de interferencia se centran en la descripción de los niveles léxico, morfológico y sintáctico, siendo mucho menos numerosos los que se proponen analizar las características fonéticas del español en contacto con el catalán. Además, como se expondrá a continuación, son contadísimos los que realizan análisis acústicos que permitan precisar las propiedades de las distintas realizaciones fónicas consideradas.

Entre las observaciones de carácter auditivo realizadas sobre la pronunciación del español de Cataluña por hablantes bilingües cabe señalar, en primer lugar, las de Badía Margarit (1979: 148-149): “[... no és gens estrany que, en medis populars catalans, hom no distingeixi entre 's' i ' $z$ ', 
en parlar castellà, i digui: 'el sielo assul' (en lloc de: 'el cielo azul') [...] la pronunciació de la 'l', que té en català una ressonància velar forta (que la fa semblant a la de l'anglès), i que, incorporada per la majoria de catalans a llur pronunciació del castellà, tant enlletgeix la dicció que en resulta. Hem constatat diverses vegades que molts universitaris catalans no s'adonen de la diferencia existent entre les dues modalitats de 'l' (catalana i castellana) [...] un hom comprèn per què la 'l' velar és tan emprada per gent cultivada quan s'expressen en castellà [...] El català sonoritza la " s' final davant la vocal inicial del mot següent, en virtut del fenòmen dit de la 'liaison'. I això mateix fan els catalans en llur castellà parlat: 'los árboles' (pronunciat, a la catalana, 'lozárboles'), 'los hombres' (pronunciat 'lozómbres'), i àdhuc 'deshacer' pronunciat 'dezacér') [...] els catalans, en parlar castellà, obren massa les vocals intermèdies del castellà, i pronuncien la seva 'e' normal com la 'e' oberta del català, i, semblantment, la seva 'o' normal com la 'o' oberta del català [...] Els catalans també apliquen llur sistema vocàlic inaccentuat a la pronunciació del castellà [...]”. Como puede apreciarse, Badía Margarit señala los principales fenómenos que son fácilmente detectables en el habla de los catalanohablantes cuando se expresan en español y sus observaciones sobre la abertura de las vocales son reproducidas por los demás autores que se interesan por dicha cuestión.

Casanovas (1995) estudia, también auditivamente, la interferencia fonética en el español de Lérida y aporta las siguientes observaciones (págs.

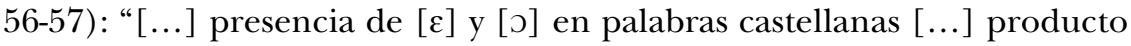
de la permanencia de los dos fonemas catalanes [...] En el orden interdental se aprecia, en primer lugar, la articulación ensordecida de /d/ implosiva, convirtiéndose, prácticamente en [t], como 'pared' [parét] [...] la articulación $[\delta]$ se mantiene en cualquier contexto, incluso en participios [...] Se conserva del mismo modo la pronunciación culta de los grupos consonánticos. Así ‘acción’ es [ak $\theta$ jón] y ‘examen', [eksámen], sin la relajación propia de las áreas hispanohablantes [...] es destacable la pronunciación velarizada de /1/ en cualquier contexto, que será más acusada cuanto más velarizada esté la realización en catalán [...] la sonorización de /s/ por fonética sintáctica [...]". Para esta autora, la presencia de vocales medias abiertas en el español de los hablantes de Lérida constituye también el fenómeno más representativo de la pronunciación de esta clase de segmentos.

Los pocos estudios de carácter experimental referidos a los elementos segmentales se centran en el análisis de los sonidos vocálicos y sus resultados muestran fenómenos diferentes a los señalados en las observaciones de carácter auditivo. Balari, Llisterri y Poch (1988) y Poch-Olivé y Harmegnies (1994) analizan las vocales del español realizadas por hablantes bilingües español-catalán. En el primer trabajo el corpus analizado es una lista de 
palabras leída por 10 informantes bilingües de dominancia catalana y, en el segundo, el corpus está constituido por una importante muestra de 150 palabras extraídas de una grabación de habla espontánea y leídas, después, en forma de lista por un hablante bilingüe también de clara dominancia catalana. En ambos casos, los resultados obtenidos muestran que, contrariamente a lo que indican las observaciones de carácter auditivo, son muy poco frecuentes las realizaciones abiertas de los fonemas /e/ y /o/ del español en el habla de los informantes analizados, puesto que la mayoría de los sonidos producidos son vocales claramente cerradas. Los valores medios de F1 y F2 así como las áreas de dispersión de las realizaciones estudiadas sugieren, desde el punto de vista de la conciencia fonológica, que los informantes bilingües utilizados en estos estudios eliminan las realizaciones $[\varepsilon]$ y $[\supset]$, propias del catalán, cuando se expresan en español.

Los diversos estudios de Harmegnies, Bruyninckx, Llisterri y Poch (1989), (1990) y (1992) se centran en el análisis de las variaciones experimentadas por la cualidad de voz de los hablantes bilingües al hablar una $\mathrm{u}$ otra de sus dos lenguas. La técnica experimental utilizada es el LTAS y la cuantificación de las variaciones se realiza mediante el cálculo del valor del índice de disimilaridad SDDD desarrollado por Harmegnies (1988). Estos autores analizan las producciones de dos grupos de hablantes bilingües: un grupo cuya lengua dominante es el catalán y otro grupo cuya lengua dominante es el español. Los resultados obtenidos indican que los hablantes bilingües suelen presentar mayores variaciones en su LTAS en su lengua dominante mientras que su comportamiento fónico en la lengua no dominante presenta menor variabilidad, es decir, podría considerarse "más rígido". El fenómeno podría interpretarse en términos de grados de bilingüismo, puesto que los hablantes, cuando se expresan en su lengua dominante, son capaces de introducir en sus producciones fenómenos de variabilidad mientras que no lo hacen cuando emplean su lengua no dominante, probablemente por sentirse menos seguros cuando la utilizan. Estos datos son congruentes con los estudios de Balari, Llisterri y Poch (1988) y Poch-Olivé y Harmegnies (1994), puesto que en ellos se analizan hablantes bilingües que se expresan en su lengua no dominante y no controlan adecuadamente la variabilidad de las realizaciones.

Finalmente, entre los estudios de carácter experimental debe destacarse el trabajo de Nadeu (2014) que analiza los efectos del acento y de la velocidad de habla sobre el timbre y la duración de las vocales del español y de la variedad central del catalán. La autora muestra que, en ambas lenguas, la ausencia de acento influye sobre las características del timbre vocálico y ello se manifiesta a través de la aparición de realizaciones centralizadas y de menor duración que la propia de las vocales acentuadas.

A la vista de los resultados obtenidos en los trabajos citados, el presente estudio se inserta en el marco de los trabajos experimentales y se pro- 
pone estudiar las características de las vocales del español realizadas por hablantes bilingües de catalán y español, cuyo uso de ambas lenguas es distinto, con el objetivo de verificar el peso de la lengua más utilizada en las características de las vocales pronunciadas. Así, se han tomado en consideración, como se indicará, hablantes cuyo porcentaje de utilización del español es mucho mayor que el del catalán, hablantes que presentan la situación opuesta y hablantes cuyo empleo de ambas lenguas es similar. En todos los casos se ha analizado un corpus de lista de palabras y un corpus de habla espontánea con el fin de estudiar la dinámica global a la que está sometido el sistema vocálico estudiado en función del estilo de habla en el que se expresan los informantes.

Las dos lenguas que manejan los hablantes estudiados poseen sistemas vocálicos muy diferentes. El sistema del español (RAE, 2011: 76-90) contiene cinco segmentos fonológicos (/ i e a o u /) y sus características articulatorias y acústicas dependen exclusivamente de factores de carácter fonético: grado de acentuación, estructura de la sílaba, entorno consonántico, estilo de habla, etc. El catalán (Julià, 2002: 40-53), en cambio, presenta dos subsistemas fonológicos en la variedad central que es la propia de los hablantes analizados. El subsistema tónico comprende los segmentos / i e $\varepsilon$ a $\supset \mathrm{u} /$ mientras que la reducción fonológica conduce a la existencia de un subsistema átono de tres unidades / i u a / y a la existencia de una vocal centralizada [ə], cuyo estatus fonológico es discutido por muchos autores. Así, los hablantes bilingües español/catalán manejan dos lenguas que presentan profundas diferencias fonológicas, pues, además del fenómeno de reducción, se distinguen en que mientras el español presenta tres grados de abertura, el catalán posee cuatro.

\section{Metodología}

\subsection{Informantes}

Para establecer el porcentaje de uso de las dos lenguas por parte de cada uno de los hablantes se ha diseñado, en el marco del proyecto FFI 2012-33499: El español en contacto con el catalán: variación diatópica y bilingüismo, un cuestionario de historial lingüístico cuyas preguntas sobre la utilización de cada lengua contemplan el comportamiento de los informantes en las diversas situaciones comunicativas propias de su vida cotidiana, así como su manejo de las cuatro destrezas comunicativas (comprensión oral y escrita y expresión oral y escrita) ${ }^{1}$. A continuación, se ha otorgado una puntuación a cada

\footnotetext{
${ }^{1}$ El cuestionario puede consultarse en la siguiente dirección de Internet: $<$ https://sites. google.com/site/espanolcontactocatalan/home>.
} 
ítem del cuestionario de forma que ha sido posible calcular automáticamente, en función de las respuestas, los porcentajes de utilización de cada una de las dos lenguas por parte de cada uno de los informantes que han respondido a las preguntas.

A partir de dichos porcentajes se han seleccionado 6 hablantes (todos ellos de sexo masculino) como informantes para este estudio. Dos de ellos presentan una utilización del español del $80 \%$ y del catalán del $20 \%$ y han sido elegidos como representantes de los hablantes bilingües que se expresan casi siempre en español. Otros dos presentan un uso inverso de sus dos lenguas: emplean el catalán en un $80 \%$ y el español en un $20 \%$. Así, estos hablantes pueden considerarse bilingües con una clara dominancia de una $\mathrm{u}$ otra de sus dos lenguas. Finalmente, se han escogido otros dos hablantes que se expresan en un porcentaje muy semejante en español y en catalán: entre el $40 \%$ y el $60 \%$. Se trata, en este caso, de bilingües que pueden considerarse equilibrados en el uso de las lenguas en las que se comunican. Los informantes analizados, por tanto, pueden caracterizarse de esta forma (véase figura 1):

FIGURA 1. Perfil lingüístico de los informantes analizados

\begin{tabular}{|c|c|c|l|}
\hline Uso del español & Uso del catalán & Número de hablantes & Dominancia \\
\hline $80 \%$ & $20 \%$ & 2 & ESPAÑOL \\
\hline $20 \%$ & $80 \%$ & 2 & CATALÁN \\
\hline $40-60 \%$ & $40-60 \%$ & 2 & EQUILIBRIO \\
\hline
\end{tabular}

\subsection{Corpus}

El corpus de análisis ha sido obtenido mediante el procedimiento de Harmegnies-Poch-Olivé (1992): a partir de una conversación semidirigida con cada uno de los informantes se han seleccionado, para cada segmento vocálico, 36 palabras, en 18 de las cuales la vocal se encuentra en posición tónica y, en las otras 18 palabras, en posición átona. No se han considerado palabras función (preposiciones, determinantes, etc.) debido a que, como han demostrado Krull (1989) y Aguilar, Machuca y Martínez (1991), la pertenencia a dicha categoría comporta diferencias significativas de duración y de frecuencia en las realizaciones vocálicas. La estructura de la sílaba en la que se encuentra la vocal analizada es, siempre, CV. 
Una vez obtenidas las palabras del corpus de habla espontánea se ha confeccionado con ellas una lista aleatoria que ha sido leída por cada uno de los locutores obteniendo así la realización de las mismas vocales en un estilo de habla formal. La comparación de las producciones en los dos estilos ha permitido analizar las diferencias de comportamiento fónico de los hablantes en función de dicho factor.

Se ha analizado, por tanto, un total de dos mil ciento sesenta vocales: 36 realizaciones de cada vocal en dos estilos de habla por parte de 6 informantes $(36 \times 5 \times 2 \times 6=2160)$.

\subsection{Análisis}

Las grabaciones han sido analizadas mediante el programa PRAAT (Boersma y Weenink, 2011) y se han realizado las siguientes operaciones: detección de las vocales, segmentación y obtención manual de las frecuencias de F1 y F2 de las realizaciones en el centro de la vocal. Los datos obtenidos han sido sometidos a un tratamiento estadístico mediante el programa SPSS.

\section{Resultados}

Se presentarán, en primer lugar, los datos correspondientes a las vocales procedentes de la lectura de la lista de palabras, puesto que la realización cuidada se ha considerado, tradicionalmente, una referencia y, a continuación, se analizarán los extraídos del corpus de habla espontánea.

Un primer análisis de varianza (ANOVA) en el que se han tomado los valores de F1 y F2 como variables dependientes y los hablantes como variable independiente no muestra diferencias significativas para los hablantes cuya lengua dominante es el español ( $80 \%$ de uso de dicha lengua). Los valores obtenidos son: para el F1, $\mathrm{F}_{(1,719)}=0.5$, n.s y para el F2, $\mathrm{F}_{(1,719)}=1.3$, n.s.). En el caso de los hablantes cuya lengua dominante es el catalán $(80 \%$ de uso de esta lengua), los valores tampoco muestran diferencias significativas: para el F1, $\mathrm{F}_{(1,719)}=1.3$, n.s y para el F2, $\mathrm{F}_{(1,719)}=0.1$, n.s. Por esta razón, los resultados correspondientes a estos informantes se presentarán agrupados por su dominancia lingüística: por una parte los de los dos hablantes de dominancia española y, por otra, los de los dos locutores de dominancia catalana. En cambio, los datos de los dos hablantes equilibrados (entre $40 \%-60 \%$ de uso de ambas lenguas) se presentarán individualmente, puesto que el análisis de varianza ha arrojado diferencias significativas en el F1: $\left(F_{(1,719)}=42.47, p=.000\right)$, no así para el F2, $F_{(1,719)}=0.5$, n.s. 
El valor de significación de $\mathrm{p}<.05$ nos indica que los resultados obtenidos tienen un $95 \%$ de probabilidad de que el comportamiento de los datos se deba al factor que se está analizando, por tanto, desde un punto de vista científico se acepta que si $p$ es inferior a .05 , quiere decir que las diferencias son estadísticamente significativas y que el investigador acepta un error en el comportamiento de sus datos de hasta un $5 \%$.

\subsection{Lista de palabras}

\subsubsection{Hablantes de dominancia española y catalana}

En la Figura 2 se muestran los valores de frecuencia del primer formante (F1) y las desviaciones típicas de las vocales analizadas en lectura para los hablantes de dominancia española y de dominancia catalana. La figura permite apreciar que los valores medios de las vocales de grado máximo y medio de abertura son un poco más elevados en los hablantes de mayor uso del español que en los hablantes de mayor uso del catalán. Por el contrario, las vocales de grado mínimo de abertura ofrecen unos valores del primer formante más bajos en los hablantes de dominancia española que en los de dominancia catalana. Los valores de la desviación típica son más elevados en los hablantes de dominancia española para todas las vocales, excepto para la vocal más abierta, [a], que muestra un grado mayor de

FIGURA 2. Valores medios (izquierda) y desviación estándar (derecha) de la frecuencia del primer formante (F1) de las vocales en lista de palabras según el grado de dominancia

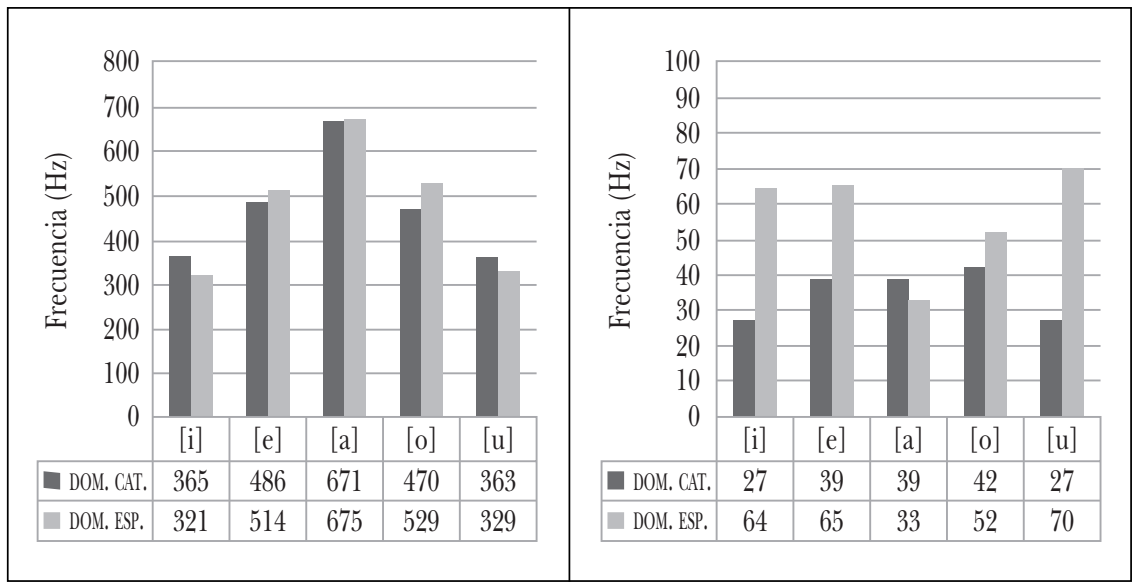


dispersión en los hablantes de dominancia catalana. Por tanto, dado que el comportamiento del F1 de las vocales está asociado a su grado de abertura, los hablantes de dominancia española presentan realizaciones más abiertas de las vocales medias que los de dominancia catalana.

Un análisis ANOVA de un factor, cuya variable dependiente es el F1 y la independiente el grado de dominancia, indica que existe una diferencia significativa en todas las vocales, excepto para la vocal [a], ya que es la única que muestra un nivel de significación por debajo de .05 , (vocal [a]: $\mathrm{F}_{(1,143)}=0.4$, n.s., vocal $[\mathrm{e}]: \mathrm{F}_{(1,143)}=9.7, \mathrm{p}=.002$, vocal $[\mathrm{i}]: \mathrm{F}_{(1,143)}=28.5$, $\mathrm{p}=.000$, vocal $[\mathrm{o}]: \mathrm{F}_{(1,143)}=53.6, \mathrm{p}=.000$, vocal $\left.[\mathrm{u}]: \mathrm{F}_{(1,143)}=14.1, \mathrm{p}=.000\right)$.

La figura 3 muestra los valores medios (izquierda) y las desviaciones típicas (derecha) del segundo formante (F2) de las vocales procedentes de la lista de palabras realizadas por los hablantes de dominancia española y catalana. Los datos presentan unos valores ligeramente más elevados en todas las vocales, excepto en la [e], para los hablantes que hacen un mayor uso del español. Los valores de la desviación típica muestran, en todos los casos, valores más altos en los hablantes de dominancia española que en los de dominancia catalana, sobre todo en las vocales [i], [o] y [u]. El análisis ANOVA, tomando en este caso el F2 como variable dependiente, indica que existe una diferencia significativa para las vocales $[\mathrm{e}, \mathrm{o}, \mathrm{u}]$ en función del grado de dominancia del hablante (vocal $[\mathrm{a}]: \mathrm{F}_{(1,143)}=2.4$, n.s., vocal $[\mathrm{e}]$ : $\mathrm{F}_{(1,143)}=7.2, \mathrm{p}=.008$, vocal $[\mathrm{i}]: \mathrm{F}_{(1,143)}=0.8$, n.s., vocal $[\mathrm{o}]: \mathrm{F}_{(1,143)}=9.08$, $\mathrm{p}=.003$, vocal $\left.[\mathrm{u}]: \mathrm{F}_{(1,143)}=12.8, \mathrm{p}=.000\right)$. Podemos afirmar, por tanto, que

Figura 3. Valores medios (izquierda) y desviación estándar (derecha) de la frecuencia del segundo formante (F2) de las vocales en lista de palabras según el grado de dominancia

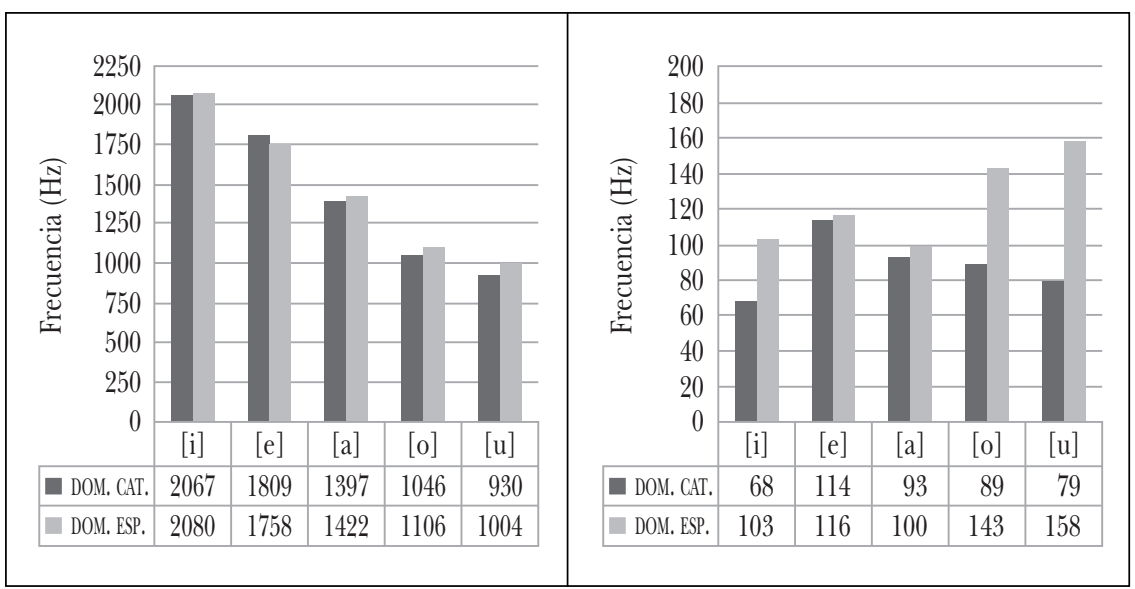


la desviación de los datos es un parámetro que sirve para diferenciar las vocales por el grado de dominancia, mientras que los valores medios de las vocales no siempre permiten establecer esta diferencia.

La figura 4 muestra las áreas de dispersión de las vocales del español en función de la dominancia de los hablantes. Las cinco áreas de dispersión correspondientes a cada una de las vocales quedan bien diferenciadas en el gráfico, como es habitual en las realizaciones de carácter formal. Es importante señalar que, tal como se ha mencionado, se observa claramente una mayor dispersión en los valores del primer y segundo formante en los hablantes de dominancia española, lo que parece sugerir que estos informantes se comportan de forma parecida a los hispanohablantes monolingües ante los fenómenos de variabilidad (Harmegnies y Poch-Olivé, 1992). Por otra parte, puede verse claramente, en la distribución de las realizaciones en el espacio vocálico, que los hablantes de dominancia catalana agrupan las realizaciones de los segmentos /e/ y /o/ del español en una zona que corresponde a las realizaciones de los fonemas /e/ y /o/ del catalán dejando una zona vacía en el lugar donde se situarían las realizaciones de los fonemas $/ \varepsilon /$ y / $/$ del catalán y las realizaciones más abiertas de /e/ y /o/ del español. En cambio, los hablantes de dominancia espa-

Figura 4. Áreas de dispersión de las vocales en lista de palabras según la dominancia de uso del español y del catalán

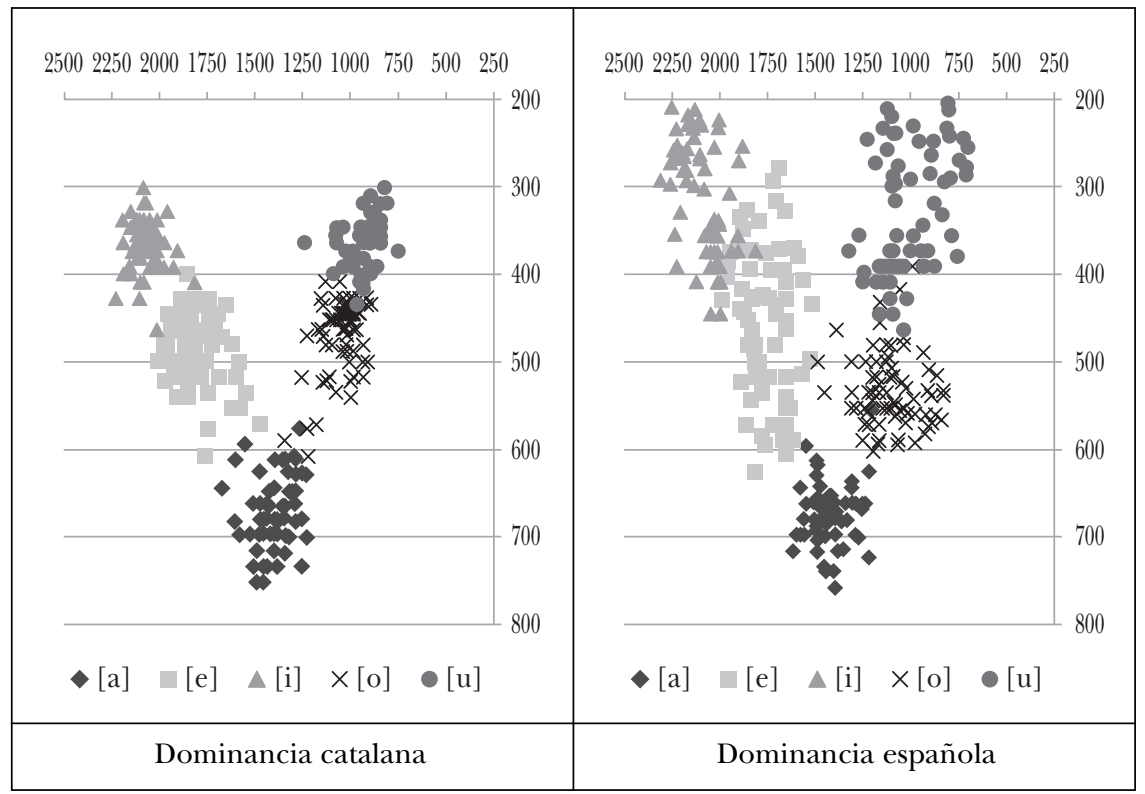


ñola sitúan sus realizaciones de /e/ y /o/ de dicha lengua distribuidas en una superficie similar a la utilizada por los hablantes monolingües.

\subsubsection{Hablantes con un uso equilibrado del español y del catalán}

Como se ha explicado, los resultados correspondientes a los informantes que presentan un uso equilibrado del español y del catalán se exponen por separado. En la Figura 5 se pueden observar los valores medios de F1 (izquierda) y los correspondientes a la desviación típica para cada uno de los hablantes (derecha). A.R. tiende a mostrar unos valores medios de los primeros formantes más altos que G.P., excepto para las vocales [o] y [a]. Los valores de las desviaciones típicas muestran que G.P. tiende a una mayor dispersión de los datos, sobre todo para la vocal abierta [a]. Es importante señalar también que dichos valores, excepto en el caso de la [a] de G.P., presentan valores más bajos que los de los hablantes de dominancia española y catalana, que se comportan de manera diferente. El análisis ANOVA de un factor, cuya variable dependiente es, en este caso, el F1 y la independiente, los dos hablantes, nos indica que existe una diferencia significativa para todas las vocales, excepto para la $[\mathrm{o}]$ (vocal $[\mathrm{a}]: \mathrm{F}_{(1,72)}=9.08$, $\mathrm{p}=.004, \operatorname{vocal}[\mathrm{e}]: \mathrm{F}_{(1,72)}=47.4, \mathrm{p}=.000$, vocal $[\mathrm{i}]: \mathrm{F}_{(1,72)}=258.7, \mathrm{p}=.000$, vocal $[\mathrm{o}]: \mathrm{F}_{(1,72)}=1.08$, n.s., vocal $\left.[\mathrm{u}]: \mathrm{F}_{(1,72)}=109.6, \mathrm{p}=.000\right)$. Eso quiere decir que los valores medios de todas las vocales, excepto los de la [o], sirven para diferenciar a los dos hablantes analizados.

FIGURA 5. Valores medios (izquierda) y desviación estándar (derecha) de la frecuencia del primer formante (F1) de las vocales en lista de palabras de los hablantes cuyo uso de las dos lenguas es equilibrado

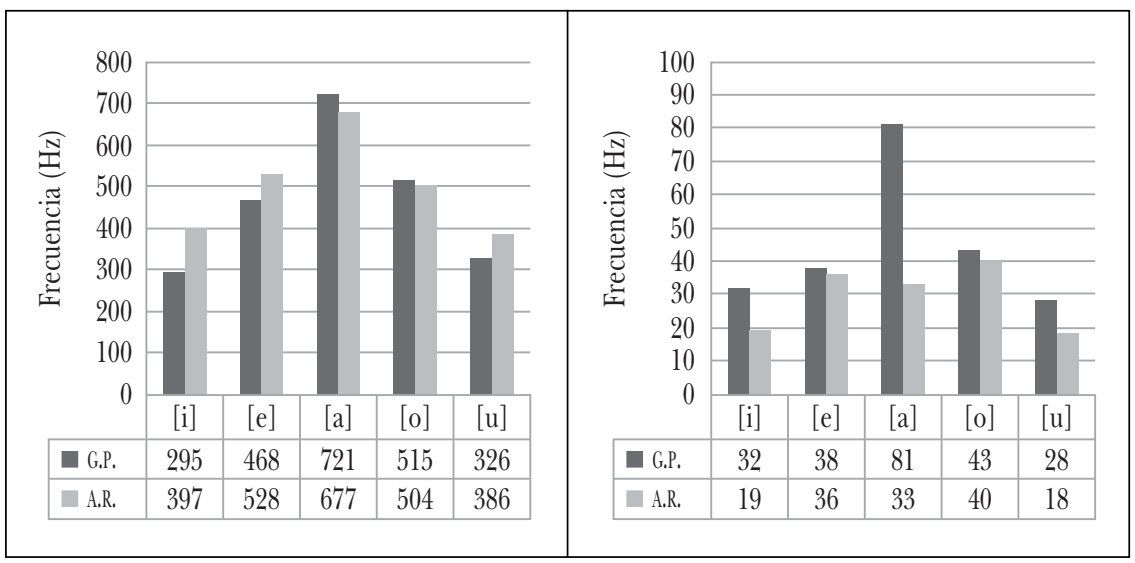


La figura 6 muestra los valores medios del segundo formante (F2) y de la desviación típica para cada uno de los hablantes equilibrados. Los datos indican que A.R. muestra generalmente valores más altos en todas las vocales; sin embargo, si se observan los datos de las desviaciones, ya no hay tanta regularidad: el hablante G.P. muestra valores de dispersión más altos para las vocales $[\mathrm{i}, \mathrm{e}, \mathrm{u}]$ y ligeramente inferiores para las vocales $[\mathrm{o}, \mathrm{a}]$. La prueba ANOVA indica diferencias significativas debidas al hablante para todas las vocales, excepto para la $[\mathrm{u}]$ (vocal $[\mathrm{a}]: \mathrm{F}_{(1,72)}=39.6, \mathrm{p}=.000$, vocal $[\mathrm{e}]: \mathrm{F}_{(1,72)}=25.5, \mathrm{p}=.000$, vocal $[\mathrm{i}]: \mathrm{F}_{(1,72)}=31.2, \mathrm{p}=.000$, vocal $[\mathrm{o}]$ : $\mathrm{F}_{(1,72)}=10,05, \mathrm{p}=.002$, vocal $[\mathrm{u}]: \mathrm{F}_{(1,72)}=1.7$, n.s.). Por tanto, se puede afirmar que, excepto para la $[\mathrm{u}]$, los valores medios de las vocales sirven para diferenciar a los dos hablantes analizados.

FiguRA 6. Valores medios (izquierda) y desviación estándar (derecha) de la frecuencia del primer formante (F2) de las vocales en lista de palabras de los hablantes cuyo uso de las dos lenguas es equilibrado

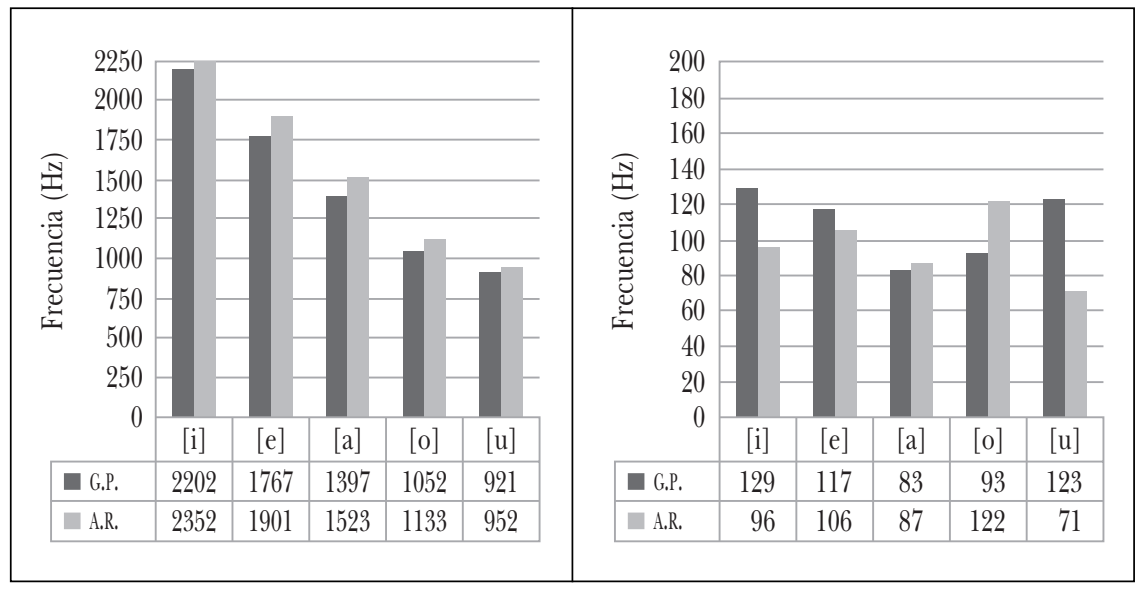

Si se comparan las áreas de dispersión de las realizaciones formales de cada uno de los hablantes equilibrados (figura 7) se puede apreciar que, en ambos casos, su comportamiento se acerca al de los monolingües hispanohablantes, así como al de los bilingües de dominancia española. Las realizaciones correspondientes a los segmentos /e/ y /o/ del español tienden a ocupar el espacio propio de estas vocales cuando son realizadas por monolingües de forma que los distintos alófonos se distribuyen en la totalidad del espacio propio de las realizaciones de los fonemas /e/ y /o/ del español. En cambio, los dos informantes muestran importantes diferencias en las características de las realizaciones de las vocales anteriores /i/ y /e/: 
FiguRA 7. Áreas de dispersión de las vocales lista de palabras de los hablantes cuyo uso de las dos lenguas es equilibrado

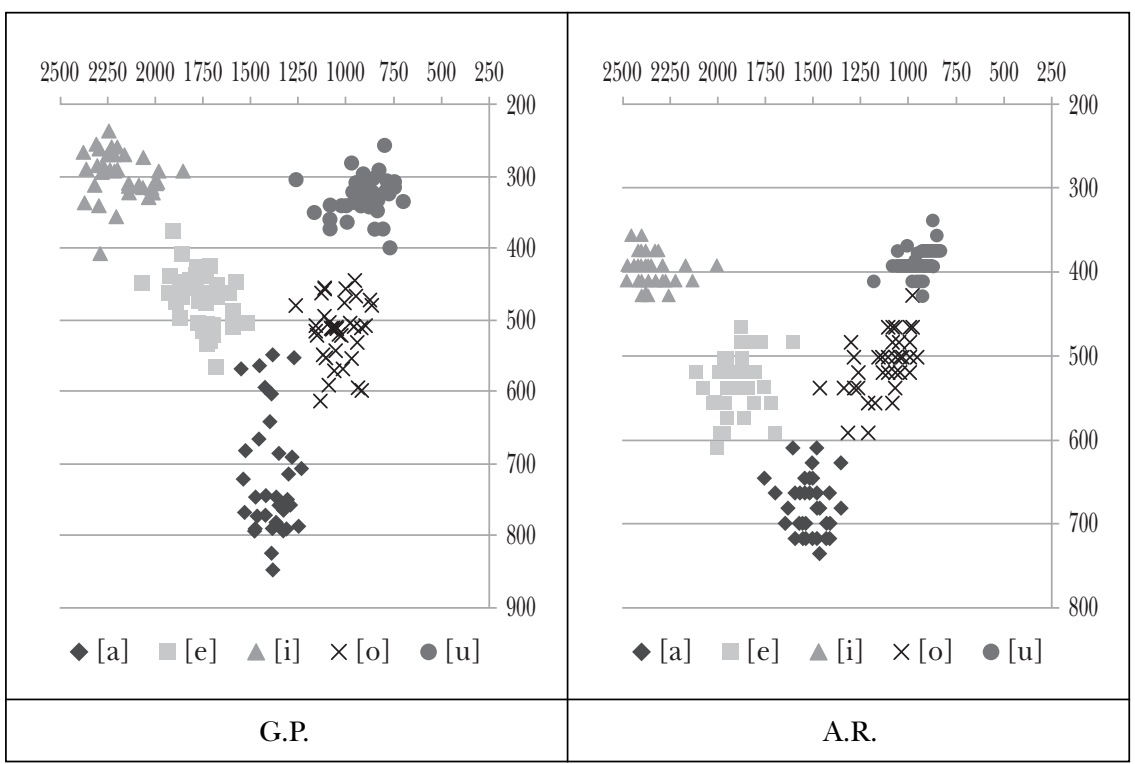

distinto grado de abertura y diferencias en el eje de anterioridad/posterioridad. Debe destacarse, también, el hecho de que las realizaciones de /a/, especialmente en el caso de G.P., tienden a desplazarse hacia el centro del espacio vocálico en el eje de abertura de forma que una parte de ellas tienden a cerrarse.

\subsection{Habla espontánea}

Los estudios realizados sobre las características de las vocales del español y del catalán en habla espontánea muestran que el cambio de estilo de habla produce un efecto de desorganización en el sistema (véase para el español, Harmegnies y Poch Olivé, 1991). El estilo informal propicia la aparición de realizaciones hipoarticuladas, menos cuidadas y menos precisas en sus propiedades que las propias de estilos formales, como la lectura de una lista de palabras, que facilita la pronunciación hiperarticulada. Se examinarán a continuación los datos obtenidos del análisis de las vocales procedentes de la conversación espontánea con el fin de establecer el tipo de dinámica que sufren las realizaciones del sistema vocálico en español por parte de hablantes bilingües. 


\subsubsection{Hablantes de dominancia española y catalana}

Los valores medios de frecuencia (izquierda) del primer formante (F1) de las vocales del corpus de habla espontánea y los correspondientes a las desviaciones típicas (derecha) de los hablantes de dominancia española y catalana pueden apreciarse en la Figura 8. Los hablantes que utilizan el catalán en un $80 \%$ presentan, en general, unos valores medios de frecuencia más altos que los que utilizan el español en un $80 \%$, excepto para la vocal $[u]$. Son muy significativos los valores de las desviaciones típicas, puesto que los hablantes de dominancia española presentan valores mucho más elevados de este índice que los que presentan los hablantes de dominancia catalana. Ello sugiere que el comportamiento de los informantes de dominancia española se asemejaría más al de los hispanohablantes monolingües que el de los informantes de dominancia catalana. La dispersión de las realizaciones es mayor para la vocal central [a] y para las vocales medias [e] y [o] que para las vocales cerradas [i] y [u]. Un análisis ANOVA de un factor, cuya variable dependiente es el F1 y la independiente el grado de dominancia, nos indica que existe una diferencia significativa en todas las vocales, excepto para las vocales $[\mathrm{o}] \mathrm{y}[\mathrm{u}]$ (vocal $[\mathrm{a}]: \mathrm{F}_{(1,143)}=11.96$, $\mathrm{p}=.001$, vocal $[\mathrm{e}]: \mathrm{F}_{(1,143)}=6.53, \mathrm{p}=.01$, vocal $[\mathrm{i}]: \mathrm{F}_{(1,143)}=6.61, \mathrm{p}=.01$, vocal $[\mathrm{o}]: \mathrm{F}_{(1,143)}=0.9$, n.s., vocal $[\mathrm{u}]: \mathrm{F}_{(1,143)}=0.6$, n.s. $)$. Por tanto, excepto para las vocales posteriores, los valores medios de las vocales determinan la realización de los hablantes en función de su grado de bilingüismo.

FIGURA 8. Valores medios (izquierda) y desviación estándar (derecha) de la frecuencia del primer formante (F1) de las vocales en habla espontánea de los hablantes de dominancia española y catalana

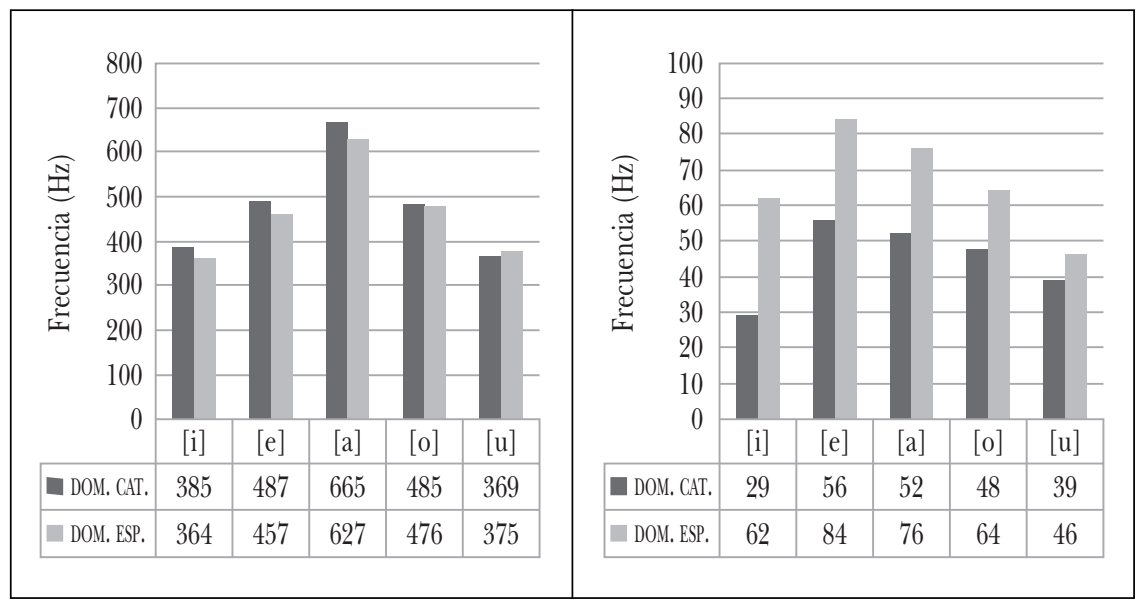


La figura 9 presenta los mismos datos, pero referidos al segundo formante (F2). Puede observarse que si bien los valores medios de frecuencia son muy semejantes en los dos informantes, no ocurre así con los valores de la desviación típica que, de nuevo, son muy superiores en el caso de los hablantes de dominancia española que en el caso de los hablantes de dominancia catalana, corroborando el comportamiento apuntado con respecto al primer formante (F1). Un análisis ANOVA de un factor, cuya variable dependiente es el F2 y la independiente el grado de dominancia, nos indica que existe una diferencia significativa solo para la vocal [u] (vocal [a]: $\mathrm{F}_{(1,143)}=2.6$, n.s., vocal $[\mathrm{e}]: \mathrm{F}_{(1,143)}=0.08$, n.s., vocal $[\mathrm{i}]: \mathrm{F}_{(1,143)}=0.67$, n.s., vocal $[\mathrm{o}]: \mathrm{F}_{(1,143)}=3.4$, n.s., vocal $\left.[\mathrm{u}]: \mathrm{F}_{(1,143)}=8, \mathrm{p}=.005\right)$, lo que quiere decir que, en general, los valores medios de las vocales no diferencian a los hablantes por su grado de bilingüismo.

FIGURA 9. Valores medios (izquierda) y desviación estándar (derecha) de la frecuencia del segundo formante (F2) de las vocales en habla espontánea de los hablantes de dominancia española y catalana

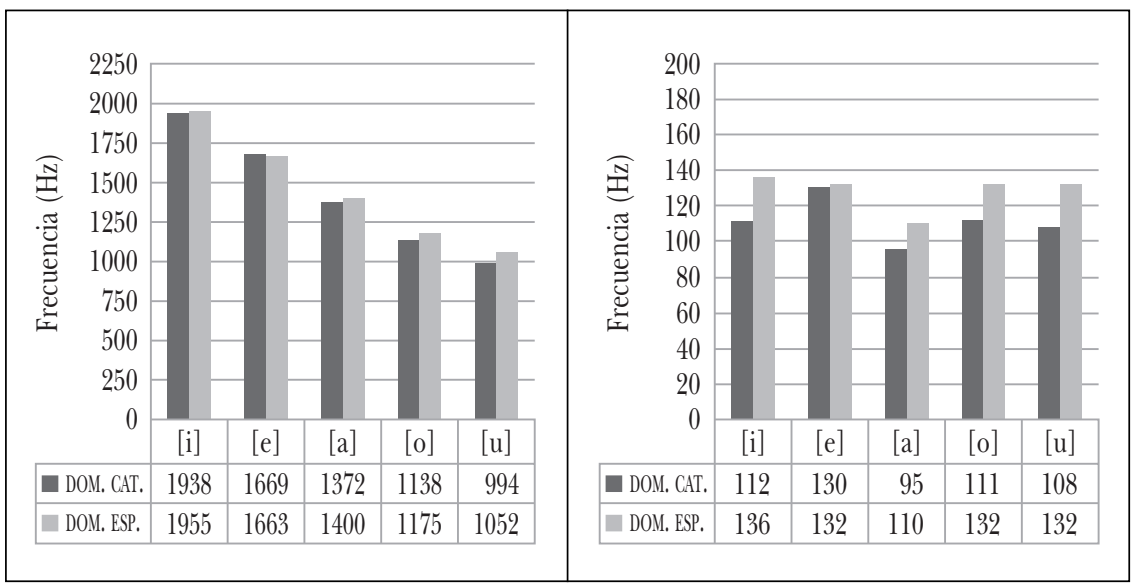

En las áreas de dispersión de estas vocales (Figura 10) se puede apreciar que la desorganización del sistema es mayor en el caso de los hablantes de dominancia española que en el caso de los hablantes de dominancia catalana, es decir, el comportamiento de los primeros es más parecido al de los hispanohablantes monolingües. Mientras los bilingües de dominancia catalana tienden a conservar una cierta estructura en sus realizaciones espontáneas en español, los de dominancia española tienden a ocupar todo el espacio vocálico invadiendo el espacio correspondiente a las realizaciones de fonemas vecinos. Debe también señalarse que el centro del espacio 
vocálico, el que ocuparían la vocal [ə] del catalán y las realizaciones relajadas de [e] [o] y [a] de los hispanohablantes monolingües, contiene numerosas realizaciones en el caso de los informantes de dominancia española, pero muchas menos en el caso de los hablantes de dominancia catalana. Se diría que una cierta conciencia fonológica del catalán ejerce una influencia importante sobre la ocupación del espacio vocálico por parte de estos informantes cuando se expresan en su lengua no dominante. Finalmente, cabe señalar que los bilingües de dominancia catalana tienden a separar las realizaciones de [a] de las propias de las vocales medias, lo cual implica una presencia menor de realizaciones abiertas de los fonemas /e/ y /o/, como ocurría también, en mayor grado, en las vocales procedentes de listas de palabras.

Figura 10. Áreas de dispersión de las vocales en habla espontánea según la dominancia de uso de las lenguas

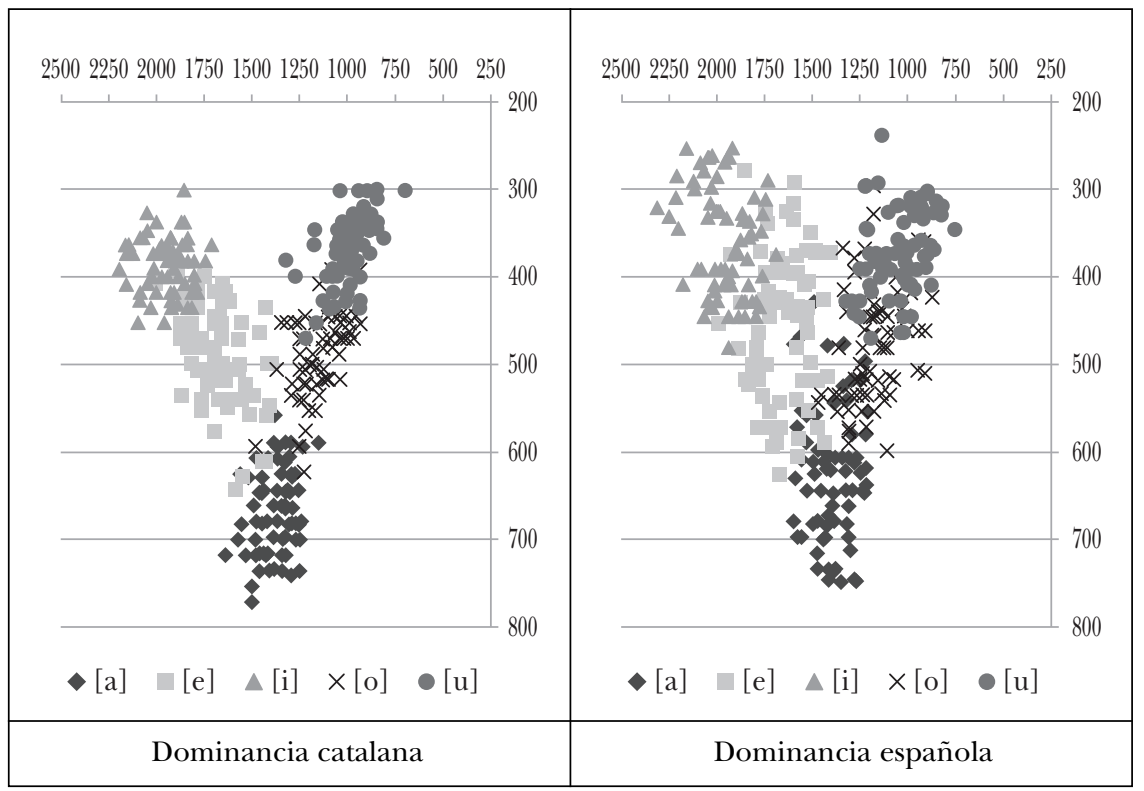

2.2.2. Hablantes con un uso equilibrado del español y del catalán

En la Figura 11 se pueden observar los valores medios de F1 (izquierda) y las desviaciones típicas (derecha) para cada uno de los hablantes cuyo uso de las dos lenguas es equilibrado. Los dos hablantes muestran las mis- 
mas tendencias que en la lectura de la lista de palabras: A.R. muestra unos valores medios de F1 más altos que G.P. y este último, según indican los valores de las desviaciones típicas, tiende a presentar una mayor dispersión de los datos. Un análisis ANOVA de un factor, cuya variable dependiente es el F1 y la independiente, los dos hablantes, nos indica que existe una diferencia significativa para todas las vocales (vocal $[\mathrm{a}]: \mathrm{F}_{(1,72)}=23.08, \mathrm{p}=.000$, vocal $[\mathrm{e}]: \mathrm{F}_{(1,72)}=92.82, \mathrm{p}=.000$, vocal $[\mathrm{i}]: \mathrm{F}_{(1,72)}=226.09, \mathrm{p}=.000$, vocal $[\mathrm{o}]: \mathrm{F}_{(1,72)}=53.90, \mathrm{p}=.000$, vocal $\left.[\mathrm{u}]: \mathrm{F}_{(1,72)}=67.02, \mathrm{p}=.000\right)$, es decir, los valores medios de las dos vocales diferencian significativamente las realizaciones de los dos hablantes.

FIGURA 11. Valores medios (izquierda) y desviación estándar (derecha) de la frecuencia del primer formante (F1) de las vocales en habla espontánea de los hablantes cuyo uso de las dos lenguas es semejante

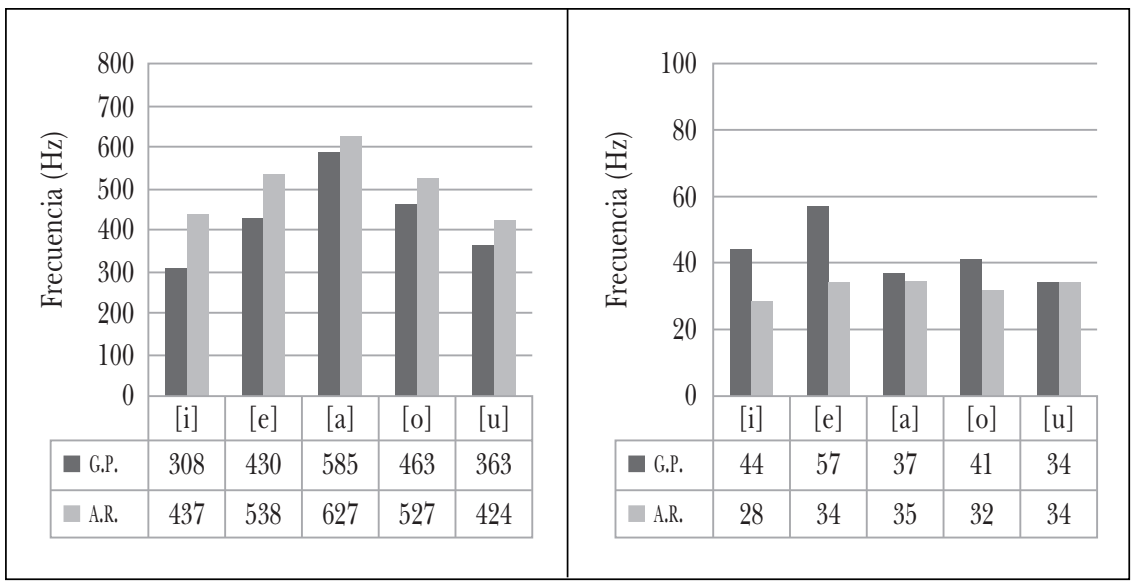

Los resultados obtenidos para el segundo formante pueden apreciarse en la Figura 12. Los datos indican que el hablante A.R. muestra generalmente valores más altos, excepto para la vocal $[u]$; pero si se observan los datos de las desviaciones, es el hablante G.P., al igual que ocurría en el primer formante, el que muestra los valores más altos. En esta ocasión, las vocales posteriores son las únicas que arrojan diferencias significativas (vocal $[\mathrm{a}]: \mathrm{F}_{(1,72)}=1.9$, n.s., vocal $[\mathrm{e}]: \mathrm{F}_{(1,72)}=3.4$, n.s., vocal $[\mathrm{i}]: \mathrm{F}_{(1,72)}=1.1$, n.s., vocal $[\mathrm{o}]: \mathrm{F}_{(1,72)}=9.9, \mathrm{p}=.002$, vocal $\left.[\mathrm{u}]: \mathrm{F}_{(1,72)}=8.4, \mathrm{p}=.005\right)$, por tanto, solo los valores medios de las vocales $[\mathrm{o}]$ y $[\mathrm{u}]$ sirven para diferenciar las realizaciones de los dos hablantes.

Si se comparan las áreas de dispersión de cada uno de los hablantes que presentan un uso similar de las lenguas analizadas (figura 13) con las de 
FIGURA 12. Valores medios (izquierda) y desviación estándar (derecha) de la frecuencia del primer formante (F2) de las vocales en habla espontánea según el grado de dominancia

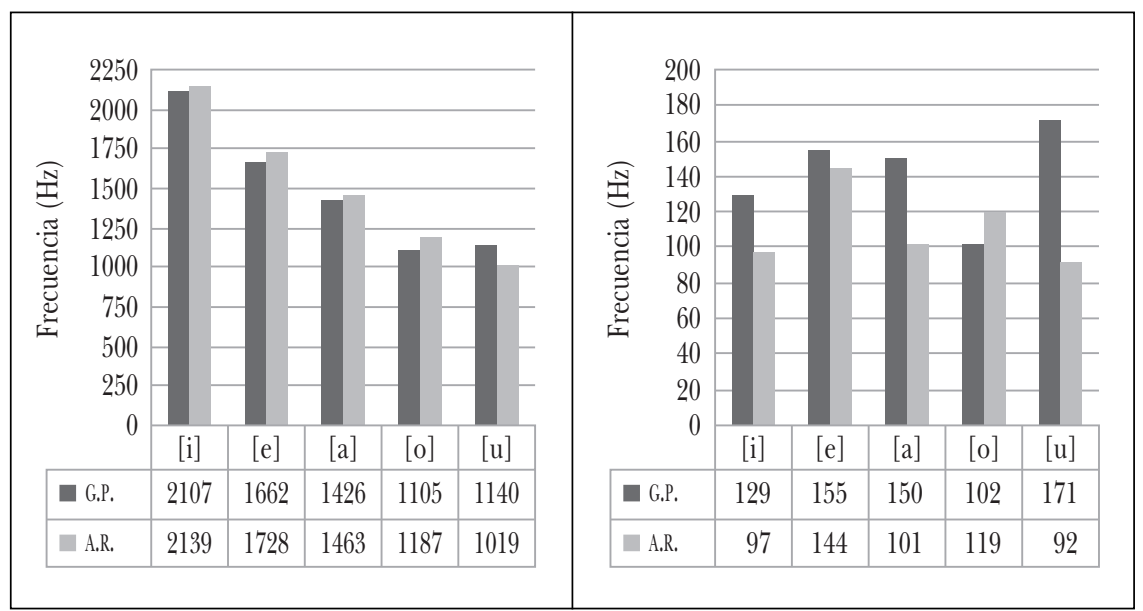

Figura 13. Áreas de dispersión de las vocales en habla espontánea de los hablantes cuya dominancia de uso es similar en las dos lenguas analizadas: catalán y español

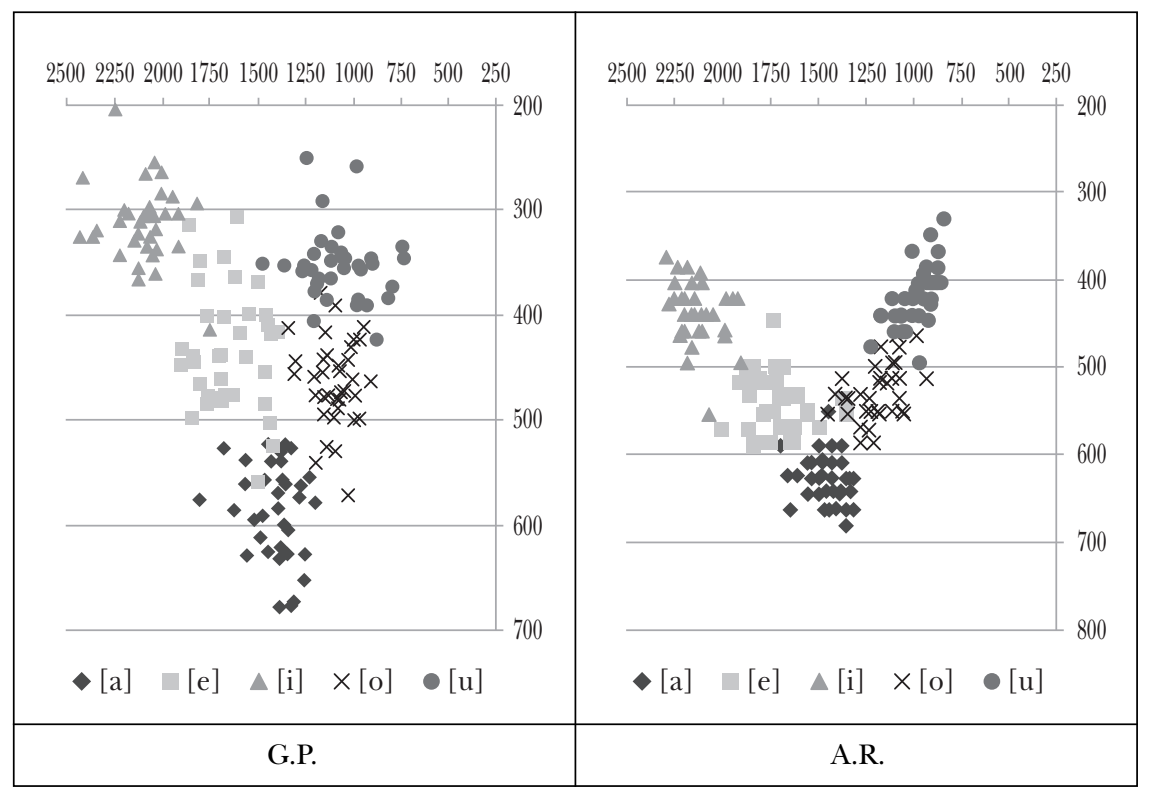


los hablantes que poseen una clara dominancia de una de las dos lenguas (véase figura 10), se puede apreciar que el informante G.P. muestra unas áreas de dispersión muy parecidas a las de los hablantes de dominancia española, en las que los valores de las vocales de grado medio de abertura tienden hacia el centro del espacio vocálico, mientras que el hablante A.R. muestra unas áreas de dispersión que se parecen más a las de los hablantes de dominancia catalana, en las que se diferencian muy bien los cinco espacios vocálicos correspondientes a cada una de las cinco vocales del español y en los que no se observa una ocupación del área central.

\section{DisCUSIÓN DE LOS RESUlTAdOS}

La interpretación adecuada de los resultados obtenidos debe tomar en consideración, en primer lugar, el comportamiento de los hablantes bilingües estudiados en relación con el propio de los hablantes monolingües de español. Los trabajos de Harmegnies y Poch Olivé (1992), Poch Olivé, Martín Butragueño y Harmegnies (2008) y Poch Olivé y Harmegnies (2010) han estudiado las características de las realizaciones vocálicas del español de hablantes monolingües españoles y mexicanos en lectura de lista de palabras y en habla espontánea, y han puesto de relieve que las áreas de dispersión se encabalgan ampliamente en habla espontánea, mientras que están claramente separadas en habla de laboratorio. Pero, simultáneamente, las áreas de dispersión son menos periféricas en habla espontánea: las vocales en habla espontánea se centralizan. Es decir, además de los factores contextuales de carácter fónico (acento, sonidos situados junto a las vocales, etc.), las características de los sonidos emitidos por los hablantes dependen de una fuente de variación de carácter global que tiende a disminuir los contrastes entre los segmentos realizados, la cual, a su vez, depende de la situación de comunicación. El grado de formalidad de la situación comunicativa determina el grado de atención que el hablante debe prestar a la pronunciación de los sonidos que emite y, por tanto, influye sobre su grado de hiper o hipo-articulación (Poch Olivé y Harmegnies, 2008: 143).

Los hablantes analizados en este trabajo, como ha podido apreciarse, muestran las mismas tendencias independientemente de su porcentaje de uso del español: en todos los casos sus realizaciones en habla espontánea se encabalgan claramente mientras que las de la lista de palabras muestran un sistema perfectamente organizado con una clara distribución de las realizaciones en las diferentes áreas. Asimismo, la tendencia a la centralización de las realizaciones está también presente en todos los hablantes, aunque, como ya se ha mencionado, aparecen en este caso algunas diferencias que pueden relacionarse con el porcentaje de uso de cada una de las lenguas por parte de los hablantes bilingües estudiados. 
Como se ha explicado, los artículos de Balari, Llisterri y Poch (1988) y Poch-Olivé y Harmegnies (1994) que estudian las vocales del español realizadas por bilingües español-catalán ponen de relieve que, contrariamente a las observaciones tradicionales de carácter auditivo, es muy escasa la frecuencia de aparición de vocales medias abiertas en el habla de estos informantes. Los seis hablantes estudiados confirman los resultados anteriores y apuntan, además, a un diferente comportamiento en función del porcentaje de uso de las dos lenguas: los dos hablantes cuyo uso del español es de un $80 \%$ (clara dominancia de dicha lengua) pronuncian las vocales del español de forma muy semejante a los monolingües, es decir, las realizaciones de las vocales medias ocupan de forma regular el espacio vocálico que se encuentra entre las vocales cerradas y la vocal abierta; los dos hablantes cuyo uso del español es de un $20 \%$ y del catalán es de un $80 \%$ (clara dominancia de la lengua catalana) tienen una clara tendencia a agrupar sus realizaciones en la zona del triángulo vocálico que correspondería a las del segmento / e/ del catalán (las vocales anteriores) y /o/ del catalán (las vocales posteriores). Dejan así un espacio vacío en las zonas que ocuparían las correspondientes realizaciones de los segmentos medios abiertos del catalán. Los dos informantes con un porcentaje de uso del español y del catalán similar (entre el $40 \%$ y el $60 \%$ ) presentan unas realizaciones cuyas características tienden a ser semejantes a las de los hispanohablantes monolingües, aunque situándose a cierta distancia de ellas, pero sin ser tampoco idénticas a las de los bilingües de dominancia catalana. Se diría que, contrariamente a la representación tradicional del bilingüe equilibrado que se siente igualmente cómodo en sus dos lenguas, se trata de hablantes que no se sienten seguros en ninguna de ellas y, por ello, su comportamiento fónico es algo errático.

\section{ConClusión}

Los resultados confirman la hipótesis de partida en el sentido de que el porcentaje de uso de cada una de las lenguas por parte de los hablantes bilingües tiene un peso importante en las características acústicas de sus realizaciones vocálicas.

Los dos hablantes cuya lengua dominante es el español presentan un comportamiento muy similar al de los hispanohablantes nativos y ello se refleja en la localización de las áreas de dispersión en el espacio vocálico. Los dos hablantes de dominancia catalana, en cambio, sitúan la mayoría de sus vocales medias en la zona del espacio vocálico que corresponde a las realizaciones cerradas de los fonemas /e/ y /o/ dejando un vacío en la zona en la que se sitúan las realizaciones abiertas de dichos fonemas en 
el caso de los hispanohablantes monolingües y de los bilingües de dominancia española. Los valores de frecuencia del primer formante están directamente relacionados con estas características de las realizaciones. Estos datos confirman los obtenidos en trabajos anteriores citados a lo largo del artículo que cuestionan las afirmaciones, a propósito de este tema, que se han realizado en estudios de carácter auditivo.

Por su parte, los dos hablantes cuyo uso de las dos lenguas es similar presentan comportamientos distintos, puesto que mientras en A.R. se observan unos valores más altos, tanto en las frecuencias del primer formante como en las del segundo, G.P. muestra una mayor dispersión en los datos. Esto indica que el estudio de este tipo de hablantes es complejo y que es necesario, en un futuro próximo, profundizar en su análisis para poder dar cuenta de las características de la pronunciación de los hablantes que utilizan dos lenguas con un porcentaje de uso similar.

Finalmente, cabe señalar que los seis hablantes estudiados se comportan de forma semejante frente al cambio de estilo de habla. Mientras las realizaciones vocálicas procedentes de la lectura de una lista de palabras se agrupan en áreas de dispersión homogéneas cuyas fronteras son claras, las procedentes de la conversación espontánea muestran un grado importante de desorganización relacionado con la relajación propia de la pronunciación en un estilo informal. En otras palabras, el porcentaje de uso de una u otra lengua ejerce mayor influencia sobre las características de las vocales que el estilo de habla en el que se expresan los hablantes. 


\section{BIBLIOGRAFÍA}

Aguilar, L., Machuca, M. J. y Martínez, G. (1991): "Analysis of the Spanish sequence 'de' in content words and in function words in continuous speech", en Proceedings of the ESCA Workshop on "Phonetics and phonology of speaking styles: reduction and elaboration in speech communication", Barcelona, págs. 7.1-7.3.

Badía Margarit, A. M. (1955): Fisiognómica comparada de las lenguas catalana y castellana. Discurso leído en la recepción pública del Dr. Don Antonio M. Badía Margarit en la Real Academia de Buenas Letras de Barcelona, Barcelona, Real Academia de Buenas Letras.

- (1965): "La integració idomàtica i cultural dels immigrants. Reflexions, fets, plans”, Qüestions de vida cristiana, 31, págs. 91-103.

- (1979): "Notes sobre el castellà parlat per catalans", en Llengua i cultura als països catalans, Barcelona, Edicions 62.

BALARI, S., LlisterRi, J. y POCH, D. (1988-1989): "La estructuración fonética de la materia sonora en hablantes bilingües”, ELUA, 5, págs. 93-98.

Boersma, P. y Weenink, D. (2011): Praat. Doing Phonetics by Computer, Ámsterdam, University of Amsterdam (<http://www.praat.org $>$ ).

Bruyninckx, M., Harmegnies, B., Llisterri, J. y Poch, D. (1990): "Bilinguisme et qualité vocale. Contribution à l'analyse des variations du spectre à long terme sous l'effet du changement de langue", en A. Landercy (ed.), Mélanges de phonétique et didactique des langues. Hommage au professeur Renard, Mons, Didier, págs. 43-53.

- (1992): "Language-induced voice quality variability in bilinguals", Journal of Phonetics, 22, págs. 19-31.

Casanovas, M. (1995): "La interferencia fonética en el español de Lleida: algunos apuntes para su estudio", Sintagma, 7, págs. 53-59.

CERDÀ, R. (1984): "Comentarios en torno a la influencia léxica del castellano sobre el catalán actual”, Beiträge zur Romanischen Philologie, 23, 2 págs. 275-281.

Colón DomÉNECH, G. (1967): "Elementos constitutivos del español: catalanismos", en Manuel Alvar et al. (eds.), Enciclopedia Lingüistica Hispánica. Vol. 2: Elementos constitutivos, fuentes, Madrid, CSIC, págs. 193-238.

HaRmegnies, B. (1988): "SDDD, a new dissimilarity index for the comparison of speech spectra”, Pattern Recognition Letters, 8, págs. 153-158.

—, Bruyninckx, M., Llisterri, J. y Poch, D. (1990): "Bilinguisme et qualité vocale. Contribution à l'analyse des variations du spectre à long terme sous l'effet du changement de langue", en A. Landercy (ed.), Melanges de phonétique et didactique des langues. Hommage au professeur Renard, Mons, Presses Universitaires de Mons/Didier Erudition, págs. 43-53.

— y Poch-Olıvé, D. (1992): "A study of style-induced vowel variability. Laboratory versus spontaneous speech in spanish", Speech Communication, 11, págs. 429-437. 
—, Bruyninckx, M., LlisterRi, J. y Poch, D. (1989): "Effects of language change ob voice quality. An experimental contribution to the study of the CatalanCastilian case", Eurospeech 1989. Proceedings of the 1st European Conference on Speech Communication and Technology, vol. 2, París, págs. 489-492.

Julià, J. (2002): "Els sons del català", en J. Solà, M. R. Lloret, J. Mascaró y M. Pérez Saldanya (dirs.), Gramàtica del català contemporani, vol. I, Barcelona, Empúries, págs. 37-87.

KRULL, D. (1989): "Consonant-vowel coarticulation in spontaneous speech and in reference words", Speech Transmission Laboratory Quarterly Progress Status Report, vol. 1, Royal Institute of Technology, Estocolmo, Suecia, págs. 101-105.

Llisterri, J., Poch, D., Harmegnies, B. y Bruyninckx, M. (1992): "Bilingüismo y calidad de voz”, en F. Etxeberria y J. Arzamendi (eds.), Bilingüismo y adquisición de lenguas, Actas del IX Congreso Nacional de AESLA, Bilbao, Universidad del País Vasco, págs. 409-416.

MARSÁ, F. (1986): "Sobre concurrencia lingüística en Cataluña”, en V. García de la Concha (ed.), El castellano actual en las comunidades bilingües de España, Salamanca, Junta de Castilla y León, Consejería de Educación y Cultura, págs. 93-104.

Moll, F. de B. (1961): "El castellano en Mallorca", en Homenaje ofrecido a Dámaso Alonso por sus amigos y discípulos con ocasión de su $60^{\circ}$ aniversario. Vol. 2. Madrid, Gredos, págs. 469-474.

NADEU, M. (2014): "Stress- and speech rate-induced vowel quality variation in Catalan and Spanish", Journal of Phonetics, 46, págs. 1-22.

PAYRATÓ, L. (1985): La interferència lingüística. Comentaris i exemples català-castellà, Barcelona, Curial/Abadia de Montserrat.

Poch-Olivé, D. y Harmegnies, B. (1994): "Dinámica de los sistemas vocálicos y bilingüismo”, Contextos, XII/23-24, págs. 7-39.

- (2010): "Centralización y reducción en las lenguas románicas", Actes du XXVe Congrès Internationale de Linguistique et de Philologie Romanes, Innsbruck, De Gruyter, págs. 137-146.

- y Martín Butragueño, P. (2008): "Influencia del estilo de habla sobre las características de las realizaciones en el español de la Ciudad de México", Actas del XV Congreso Internacional ALFAL, Montevideo, Uruguay, 18-21 de agosto de 2008, formato CD.

Real ACADEMIa Española (2011): Nueva gramática de la lengua española: fonética y fonología, vol. 3, Madrid, Espasa.

Sinner, C. (2004): El castellano de Cataluña, Tubinga, Max Niemeyer.

- y Wesch, A. (2008): "El castellano en tierras de lengua catalana: estado de la cuestión”, en C. Sinner y A. Wesch (eds.), El castellano en las tierras de habla catalana, Madrid, Frankfurt, Iberoamericana, Vervuert, págs. 11-57. 